\title{
Heterogeneity of chemosensitivity in esophageal cancer using ATP-tumor chemosensitivity assay
}

\author{
Zhi-qiang LING ${ }^{1}$, Chun-jian $\mathrm{QI}^{2}$, Xiao-xiao LU¹, Li-juan QIAN ${ }^{1}$, Lin-hui GU ${ }^{1}$, Zhi-guo ZHENG ${ }^{1}$, Qiang ZHAO ${ }^{3}$, Shi WANG ${ }^{4}$, Xian- \\ hua FANG ${ }^{5}$, Zhi-xing YANG ${ }^{6}$, Jian $\mathrm{YIN}^{7}$, Wei-min $\mathrm{MAO}^{1,3, *}$ \\ ${ }^{1}$ Zhejiang Cancer Research Institute, ${ }^{3}$ Department of Tumor Surgery, ${ }^{4}$ Department of Endoscopy, ${ }^{5}$ Department of Pathology, Zhejiang \\ Province Cancer Hospital, Zhejiang Cancer Center, Hangzhou 310022, China; ${ }^{2}$ Department of Oncology, the Changzhou No 2 People's \\ Hospital, Affiliated to Nanjing Medical University, Changzhou 213003, China; ${ }^{6}$ Institute for Nutritional Sciences, Shanghai Institute for \\ Biological Sciences, Chinese Academy of Sciences, Shanghai 200031, China; ${ }^{7}$ Huzhou Haichuang Biological Scientific Technology Co, \\ Ltd, Huzhou 313000, China
}

\begin{abstract}
Aim: Current chemotherapy for esophageal cancer is conducted on the basis of empirical information from clinical trials, which fails to take into account the known heterogeneity of chemosensitivity between patients. This study was aimed to demonstrate the degree of heterogeneity of chemosensitivity in esophageal cancers.

Methods: A total of 42 esophageal cancer specimens were collected. The heterogeneity of chemosensitivity in esophageal cancer specimens was examined using an ex vivo ATP-tumor chemosensitivity assay (ATP-TCA).

Results: Thirty eight specimens produced evaluable results (90.5\%). The most active single agent tested was nedaplatin, to which $28.9 \%$ of samples were sensitive. Combinations of chemotherapy agents exhibited much higher sensitivity: cisplatin+paclitaxel was sensitive in 16 of 38 (42.1\%) of samples, while nedaplatin+paclitaxel was more effective, which was sensitive in 20 of 38 cases (52.6\%).

Conclusion: There was a marked heterogeneity of chemosensitivity in esophageal cancer. Chemosensitivity testing may provide a practical method for testing new regimens before clinical trials in esophageal cancer patients.
\end{abstract}

Keywords: esophageal cancer; chemotherapy; heterogeneity; nedaplatin; cisplatin; paclitaxel; ATP-tumor chemosensitivity assay

Acta Pharmacologica Sinica(2012) 33: 401-406; doi: 10.1038/aps.2011.195; published online 30 Jan 2012

\section{Introduction}

Esophageal cancer is a highly malignant gastrointestinal cancer that readily progresses to widespread metastasis to lymph nodes and easily infiltrates the trachea and great vessels $^{[1]}$. Better treatment outcomes of esophageal cancer have been obtained by improved diagnostic technologies such as dye-spraying endoscopy ${ }^{[2]}$, surgical skills such as threefield lymphadenectomy ${ }^{[3]}$, and perioperative management ${ }^{[4]}$. Because esophageal cancer is generally more sensitive to anticancer drugs than other gastrointestinal carcinomas, various multidisciplinary treatments have been attempted and chemoradiation has long been established as a standard treatment for esophageal cancer because it is highly effective and can be performed relatively safely ${ }^{[5]}$. However, as in the case of surgery, chemoradiation mainly provides localized treatment,

\footnotetext{
* To whom correspondence should be addressed.

E-mail maowm1218@163.com

Received 2011-08-03 Accepted 2011-12-09
}

and treatment outcomes of cases for which radical surgery is impossible, such as cancer infiltrating into other organs, distant lymph node metastasis and metastasis to other organs, are still poor. In these cases, systemic chemotherapy is usually adopted, and many regimens have been used ${ }^{[5,6]}$.

Tumors show heterogeneity of genotype and phenotype, and such heterogeneity in esophageal cancer certainly affected response to cytotoxic agents ${ }^{[7-9]}$. Predictive assays based on thymidylate synthase levels show some promise, but cellular assays have largely been ignored due to low evaluability rates and technical problems which is common in tumor-derived tissue $^{[10]}$. However, recent technical developments have produced assays, such as the ATP-tumor chemosensitivity assay (ATP-TCA), which has high availability rates with solid tumors and produces interpretable results in more than $90 \%$ of tumors tested ${ }^{[11,12]}$. The results correlated well with outcome in patients with a sensitivity of $95 \%$ for predicting those who responded to primary treatment of stage III ovarian cancer ${ }^{[13]}$. The use of this assay was shown to double progression-free 
survival and overall survival in a case-control intervention study in recurrent ovarian carcinoma ${ }^{[14]}$.

We performed this study to determine the degree of heterogeneity of chemosensitivity in esophageal cancer as a prelude to studies of the molecular basis of resistance in tumor-derived cells and the potential use of this assay to guide therapy. We also wanted to solve the ATP-TCA technical problems, particularly the use of tumor material from different origins.

\section{Materials and methods}

All procedures complied with the ethical guidelines for the collect of human tissue specimens and use of laboratory study at Zhejiang Province Cancer Hospital, Zhejiang Cancer Center, China.

\section{Tumor specimens}

A total of 42 specimens were studied and 38 of these produced evaluable results $(90.5 \%)$. Thirty-five samples were from patients undergoing resection of their primary esophageal cancer (of all pathological stages) and three were pleural aspirates in patients with metastatic disease. The median age of the patients was 57 years (range 30-82). Local ethics committee approval was obtained and informed consent gained from all patients. Biopsies were taken from the luminal surface of resection specimens by a pathologist or surgeon, ensuring histopathological diagnosis and staging were not compromised.

\section{ATP-tumor chemosensitivity assay (ATP-TCA)}

Chemosensitivity was assessed in primary esophageal cancer tumor tissue samples using the ATP-TCA (TCA-100; DCS Innovative Diagnostik Systeme, Hamburg, Germany), which has been described in detail ${ }^{[15]}$. Briefly, surgical biopsies $\left(1-2 \mathrm{~cm}^{3}\right)$ were obtained during primary surgery. Tumor cells were isolated by mechanical and enzymatic dissociation (TDE DCS) (Innovative Diagnostik Systeme; or collagenase, Sigma, St Louis, MO, USA). Approximately $2 \times 10^{4}$ cells were then seeded into each well of a 96-well polypropylene microplate. Test drug concentrations were used in triplicate at six different doses of $6.25 \%, 12.5 \%, 25 \%, 50 \%, 100 \%$, and $200 \%$ of the test drug concentration (TDC). The TDCs were based on pharmacokinetic data for standard doses of the agents, adjusted to give good discrimination (Table 1). Two rows on each plate were reserved for controls, one row of maximum inhibitor (MI

Table 1. Drugs tested and their $100 \%$ TDC as used in the ex vivo ATP-TCA.

\begin{tabular}{lc}
\hline Drug/combination & $100 \%$ TDC $(\mu \mathrm{g} / \mathrm{mL})$ \\
\hline Paclitaxel (PTX) & 13.8 \\
Adriamycin (ADM) & 1 \\
5-Fluorouracil (5-Fu) & 25 \\
Nedaplatin (NDP) & 18 \\
Cisplatin (DDP) & 6.3 \\
DDP+PTX & $6.3 \pm 13.8$ \\
NDP+PTX & $18 \pm 13.8$ \\
DDP+5-Fu & $6.3 \pm 25$ \\
\hline
\end{tabular}

DCS) and one row of CAM only (MO-no drug control). Following preparation of the drug dilutions, $100 \mu \mathrm{L}$ of the cell suspension was added to each well of the plate. The plate was incubated for $5-6 \mathrm{~d}$ at $37^{\circ} \mathrm{C}$ with high humidity and $5 \% \mathrm{CO}_{2}$. The cells were observed every $24 \mathrm{~h}$ microscopically to check for overgrowth or infection. At the end of the incubation period the cells were lysed by the addition of $50 \mu \mathrm{L}$ of tumour cell extraction reagent (TCER DCS), and the ATP content of each well was assessed by the addition of $50 \mu \mathrm{L}$ luciferinluciferase reagent (DCS) to $50 \mu \mathrm{L}$ of extracted cells. Luminescence measurements were made using Orion II luminometer (Berthold Diagnostic Systems).

\section{Data analysis}

Data was transferred directly from the luminometer to a spreadsheet (Excel 2003; Microsoft). A TCA index, or index of sensitivity, calculated as [600-sum (inhibition $6.25 \%$ $200 \%$ )] has been shown to allow simple comparison of results between drugs and tumors. In addition, $\mathrm{IC}_{50}$ and $\mathrm{IC}_{90}$ were determined by linear interpolation. Four categories of ex vivo sensitivity were defined as: (a) strong sensitivity, $\mathrm{IC}_{90} \leq 100 \%$ TDC and $\mathrm{IC}_{50}<25 \%$ TDC; (b) partial sensitivity, $\mathrm{IC}_{90}>100 \%$ TDC and $\mathrm{IC}_{50} \leq 25 \%$ TDC; (c) weak sensitivity, $\mathrm{IC}_{90} \leq 100 \%$ TDC and $\mathrm{IC}_{50}>25 \% \mathrm{TDC}$; and (d) resistance, $\mathrm{IC}_{90}>100 \% \mathrm{TDC}$ and $\mathrm{IC}_{50}>25 \% \mathrm{TDC}$.

All experiments were performed three times and judged acceptable if the results showed a coefficient of variation below $25 \%$. The results of each experiment were entered into an access database for further analysis and compared with existing data for tumor-derived cells using descriptive statistics. Further statistical tests (SPSS Software, IL, USA) were performed when direct comparisons were necessary: the Wilcoxon rank-sum test was used to compare paired series. Combination effects were assessed using Chou's method ${ }^{[16]}$, as previously used with the ATP-TCA ${ }^{[17]}$. The combination index (CI) was determined at $90 \%$ cell death, and was defined as follows: $C_{A+B}=\left[\left(D_{A / A+B}\right) / D_{A}\right]+\left[\left(D_{B / A+B}\right) / D_{B}\right]+[$ alpha $\left.\left(D_{A / A+B} \times D_{B / A+B}\right) / D_{A} D_{B}\right]$, where $C I_{A+B}=C I$ for a fixed effect $(\mathrm{F}=90 \%)$ for the combination of cytotoxic $\mathrm{A}$ and cytotoxic $B ; D_{A / A+B}$ or $D_{B / A+B}=$ concentration of cytotoxic $A$ or $B$ in the combination $\mathrm{A}+\mathrm{B} ; \mathrm{D}_{\mathrm{A}}$ or $\mathrm{D}_{\mathrm{B}}=$ concentration of cytotoxic $\mathrm{A}$ or $\mathrm{B}$ alone; alpha $=$ parameter with value 0 when $\mathrm{A}$ and $\mathrm{B}$ are mutually exclusive, and 1 when $A$ and $B$ are mutually nonexclusive. The combination index indicated: synergism $<0.8$; additivity $>0.8$ and $<1.2$; antagonism $>1.2$; slight synergistic and additive cytotoxic activity for value of 0.8 and 1.2 , respectively.

\section{Results}

For comparison between drugs and tumors, an Index $<300$, representing an average $50 \%$ inhibition across all concentrations tested was used indicate sensitivity, as previously published $^{[12,18]}$. The results showed considerable heterogeneity of chemosensitivity to single agents and drug combinations between the tumors tested (Figure 1 and Table 2). The most active single agent tested was NDP, to which $28.95 \%$ of samples were sensitive $(P<0.05)$. Both drug combinations 

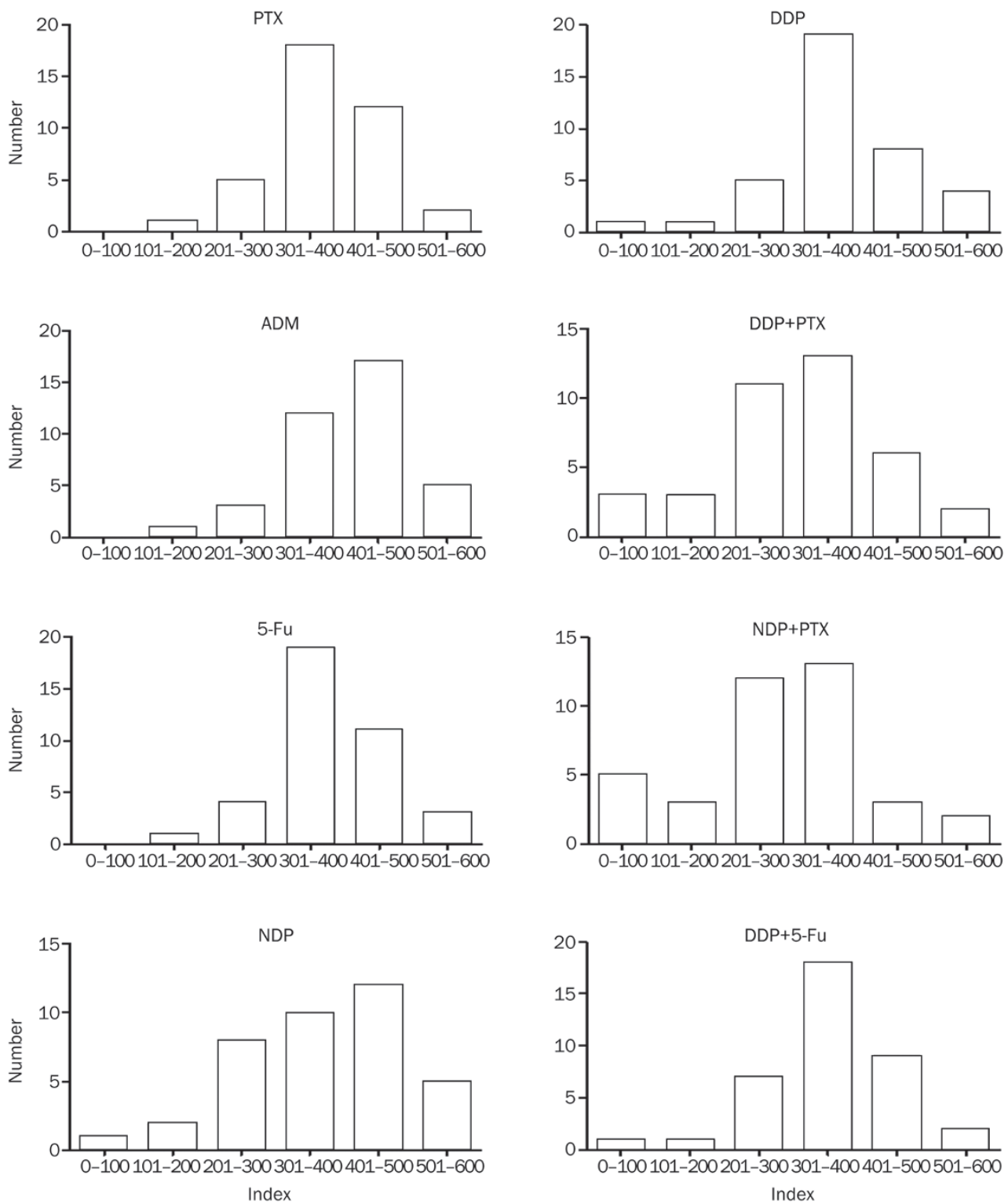

Figure 1. Frequency histograms show heterogeneity of the sensitivity index for each single agent and combination.

Table 2. Summary of sensitivity data (using an arbitrary threshold of sensitivity defined as a TCA index $<300$ for six concentrations use).

\begin{tabular}{lccc}
\hline $\begin{array}{c}\text { Drug/ } \\
\text { combination }\end{array}$ & $\begin{array}{c}\text { No } \\
\text { sensitive }\end{array}$ & $\begin{array}{c}\text { No in } \\
\text { ATP-TCA }\end{array}$ & $\begin{array}{c}\text { Sensitivity } \\
\text { assessed (\%) }\end{array}$ \\
\hline PTX & 6 & 38 & 15.8 \\
ADM & 4 & 38 & 10.5 \\
5-Fu & 5 & 38 & 13.2 \\
NDP & 11 & 38 & 29.0 \\
DDP & 7 & 38 & 18.4 \\
DDP+PTX & 16 & 38 & 42.1 \\
NDP+PTX & 20 & 38 & 52.6 \\
DDP+5-Fu & 9 & 38 & 23.7 \\
\hline
\end{tabular}

achieved greater growth inhibition than drugs used alone $(P<0.05)$, except for NDP. The correlation analysis was done using Pearson's rank correlation test among all 5 drugs tested. Results showed that there exist positive correlation among all 5 drugs tested (Figure 2 and Table 3 ).

Some tumors responded well to one drug or combination, while others showed no response to this and instead responded to an alternative regimen. For a limited panel of drugs and combinations, four cases were sensitive to only on drug/combination and resistant to all the others tested. Of these four, one was sensitive only to NDP, one to PTX, and two to NDP+PTX. One case was resistant to all drugs/combinations tested $(2.6 \%)$.

Despite appearing sensitive to certain drugs using the Index threshold of $<300$, many tumors did not reach strong sensitivity level. Table 4 showed the patterns of chemosensitivity for different agents on tumors. Again, the most active single agent was NDP. NDP alone showed a strong sensitivity in 11 of 38 tumor samples tested, but ADM was 4 of $38(29.0 \%$ vs $10.5 \%, P<0.01)$. Combinations of agents also showed more 


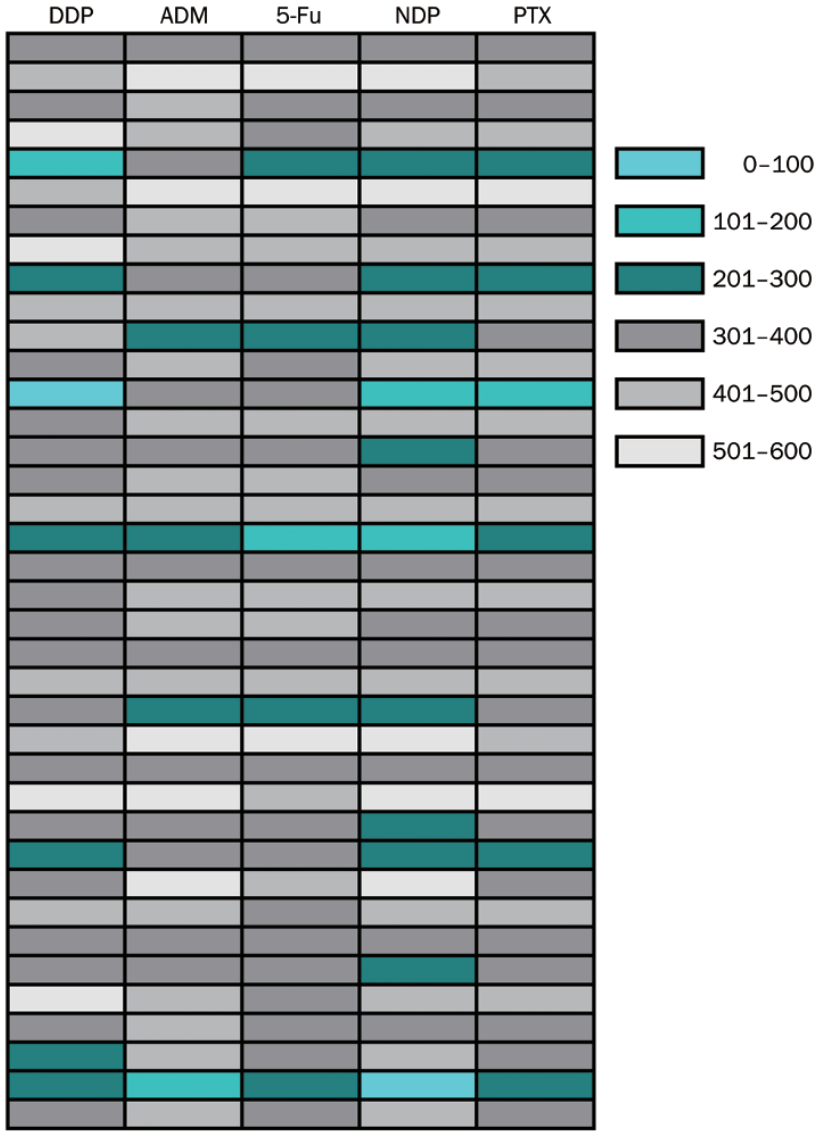

Figure 2. The ATP-TCA results of the 38 tumor specimens with five drugs were classified into 6 groups by different index values, and were marked with turquoise, cyan, dark cyan, dark grey, grey, and light grey which represent <100, 100-200, 200-300, 300-400, 400-500, and >500, respectively.

strong sensitivity cases. The DDP+PTX demonstrated a strong sensitivity in 16 of 38 of samples. The NDP+PTX was more effective, with strong sensitivity in 20 of 38 cases tested $(42.1 \%$ vs $52.6 \%, P<0.05)$.

Figure 3 showed the results of testing DDP and PTX, alone and in combination, on esophageal cancer cells. DDP dem-
Table 4. Patterns of chemosensitivity exhibited by esophageal cancer specimens.

\begin{tabular}{lcccc}
\hline $\begin{array}{c}\text { Drug/ } \\
\text { combination }\end{array}$ & $\begin{array}{c}\text { Strong } \\
\text { sensitivity }\end{array}$ & $\begin{array}{c}\text { Partial } \\
\text { sensitivity }\end{array}$ & $\begin{array}{c}\text { Weak } \\
\text { sensitivity }\end{array}$ & $\begin{array}{c}\text { Resis- } \\
\text { tance }\end{array}$ \\
\hline PTX & 9 & 5 & 7 & 17 \\
ADM & 5 & 5 & 3 & 25 \\
$5-F u$ & 7 & 11 & 3 & 17 \\
NDP & 9 & 8 & 3 & 18 \\
DDP & 8 & 11 & 3 & 16 \\
DDP+PTX & 13 & 11 & 3 & 11 \\
NDP+PTX & 18 & 11 & 1 & 8 \\
DDP+5-Fu & 10 & 13 & 4 & 11 \\
\hline
\end{tabular}

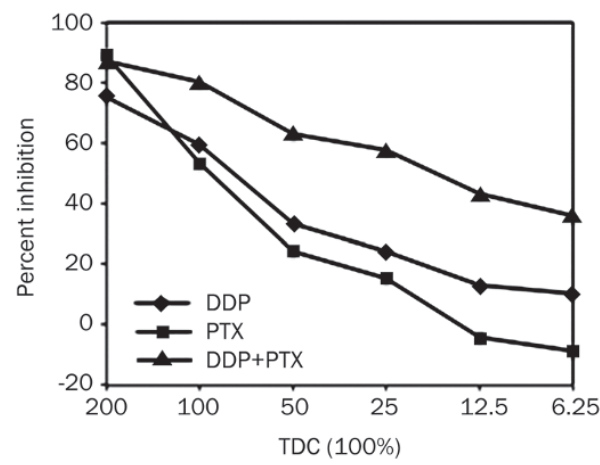

Figure 3. Results for DDP+PTX in one tumor, showing little activity of the DDP, but a synergistic increase in activity of the combination compared with PTX.

onstrated partial sensitivity on its own, but when combined with the relatively resistance PTX the sensitivity was greatly improved. DDP and PTX combination had synergistic effect $\left(\mathrm{IC}_{90}=0.75\right)$, while NDP and PTX had additive effect $\left(\mathrm{IC}_{90}=0.93\right)$ (Figure 4).

\section{Discussion}

It would be of major importance to determine appropriate drugs to be used for treatment in patients with advanced can-

Table 3. The correlation analysis using Pearson's rank correlation test among all drugs tested.

\begin{tabular}{|c|c|c|c|c|c|c|}
\hline \multicolumn{7}{|c|}{ Pearson correlation } \\
\hline & & DDP & $\mathrm{ADM}$ & $5-\mathrm{Fu}$ & NDP & PTX \\
\hline DDP & Pearson correlation & 1 & 0.569 & 0.494 & 0.745 & 0.862 \\
\hline \multirow[t]{2}{*}{ ADM } & Pearson correlation & 0.569 & 1 & 0.871 & 0.889 & 0.712 \\
\hline & $P($ 2-tailed $)$ & 0.000 & & 0.000 & 0.000 & 0.000 \\
\hline \multirow[t]{2}{*}{ NDP } & Pearson correlation & 0.745 & 0.889 & 0.793 & 1 & 0.858 \\
\hline & $P($ 2-tailed $)$ & 0.000 & 0.000 & 0.000 & & 0.000 \\
\hline \multirow[t]{2}{*}{ PTX } & Pearson correlation & 0.862 & 0.712 & 0.632 & 0.858 & 1 \\
\hline & $P(2$-tailed $)$ & 0.000 & 0.000 & 0.000 & 0.000 & \\
\hline
\end{tabular}




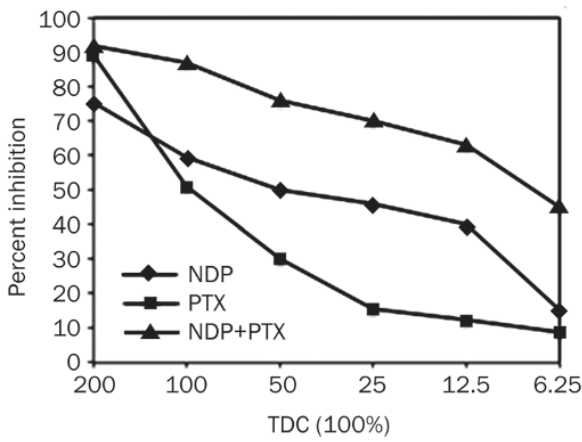

Figure 4. Results for NDP+PTX in one tumor, showing activity of the NDP, but an additive in activity of the combination compared with PTX.

cer. A number of chemosensitivity assays were developed over the last decades to predict the responsiveness of tumors to chemotherapy ${ }^{[19-21]}$. In recent years, the ATP-TCA method is a novel approach to test chemosensitivity in solid tumors ${ }^{[22-26]}$.

In present study, the availability of esophageal cancer samples using the ATP-TCA was $90.5 \%$, which is similar to the evaluability rates achieved in other 'cleaner' tumor types using this assay ${ }^{[27,28]}$. Other in vitro studies of esophageal cancer cells, including the use of the MTT assay and histoculture drug response assay, have produced similar evaluability $\operatorname{rates}^{[2,30]}$. The ATP-TCA has been shown to be more sensitive than these assays, and to have technical advantages over the MTT and clonogenic assays ${ }^{[27-30]}$. Previous studies with the ATP-TCA suggested that the assay was a good model for the investigation of tumor chemosensitivity and the results so far showed good correlation with clinical trial results in ovarian cancer ${ }^{[18,31]}$. The chemosensitivity index has been used in previous studies to differentiate between sensitive and resistant tumors. The $\mathrm{IC}_{50}$ and $\mathrm{IC}_{90}$ were particularly useful measure of the efficacy of a drug. In this study we used chemotherapeutic agents at level related to their peak plasma concentrations, taking into account their degree of protein binding. We demonstrated that $29 \%$ of tumors were sensitive to NDP with Index $<300$, and $44.7 \%$ partial sensitive, which correlated well with the clinical response rate seen in patients. The results show considerable heterogeneity of chemosensitivity between patients to single agents and to drug combinations (Figure 1). Some tumor cells responded well to particular drugs or combinations, while other tumor cells showed no response to these, but instead responded to an alternative regimen. The histograms in Figure 1 all show a bell-shaped (Gaussian-like) distribution. Other distributions, ie with 2 peaks (one peak with sensitive and a second peak with resistant tumors) or an equal distribution without peak did not occur. Hence, bell-shaped distributions seem to be a general feature for all drugs tested. In principle, 2 peaks or an equal distribution without peak, whatever is, is right. But, fundamentally, the peak characteristic depends on the degree of heterogeneity and the purity of tumor cells in ATP-TCA analysis. The correlation analysis was performed using Pearson's rank correlation test among all five drugs tested. It was found that there exist positive correlation among all five drugs tested, suggesting the tumors are crossresistant to all drugs. These also reflect the clinical reality that tumors are frequently rather cross-resistant.

Advanced esophageal cancer with widespread metastasis to lymph nodes or other organs is difficult to treat and has an extremely poor prognosis. In China, the most common chemotherapy single agent used in esophageal cancer was platinum compounds. The efficacy of platinum agents against cancer cells could be related to inhibition of DNA synthesis or to saturation of the cellular capacity to repair platinum adducts of DNA. Paclitaxel binds to tubulin and inhibits the disassembly of microtubules, thereby resulting in the inhibition of cell division $^{[32]}$. Clinical studies demonstrated a range of response rates to this regimen, most of them between $25 \%$ and $50 \%$. We tested the combinations containing NDP, which was effective to $29 \%$ tumors as a single agent. The combined treatment with NDP and PTX was the most effective group. $52.6 \%$ of samples were sensitive to adriamycin+paclitaxel using the Index $<300$ threshold and this was the most sensitive regimen (47.3\%). The adriamycin was commonly used and its clinical activity against numerous solid malignancies make it an attractive drug for use in combination therapy ${ }^{[33]}$.

In conclusion, there was a marked heterogeneity of chemosensitivity in esophageal cancer. Chemosensitivity testing might provide a practical method of testing new regimens before clinical trials in esophageal cancer patients. We believe that the ability to predict those patients who will respond well to chemotherapy will be a major step forward.

\section{Acknowledgements}

The authors wish to thank Dr Li-li ZHANG of the Huzhou Haichuang Biological Scientific Technology Co, Ltd, China for her excellent technical assistance. This work was supported in part by a grant from the Science and Technology General Project of Zhejiang Province, China (№ 2009C33143).

\section{Author contribution}

Zhi-qiang LING performed research and participated in the preparation of manuscript; Chun-jian QI contributed new analytical tools and the preparation of manuscript; Li-juan QIAN and Lin-hui GU took part in tissue culture; Xiao-xiao LU and Zhi-guo ZHENG participated in statistical analysis; Qiang ZHAO and Shi WANG collected tissue specimens; Xian-hua FANG performed pathologic diagnosis; Zhi-xing YANG analyzed the data; Jian YIN gave technical support and reagents; and Wei-min MAO designed the research.

\section{References}

1 Parkin DM, Bray F, Ferlay J, Pisani P. Estimating the world cancer burden: Globocan 2000. Int J Cancer 2001; 94: 153-6.

2 Pohl J, May A, Rabenstein T, Pech O, Nguyen-Tat M, Fissler-Eckhoff A, et al. Comparison of computed virtual chromoendoscopy and conventional chromoendoscopy with acetic acid for detection of neoplasia in Barrett's esophagus. Endoscopy 2007; 39: 594-8.

3 Gockel I, Sgourakis G, Lyros O, Hansen T, Lang H. Dissection of lymph node metastases in esophageal cancer. Expert Rev Anticancer Ther 
2011; 11: 571-8.

4 Buscaglia JM, Jayaraman V, Nagula S. Temporary use of a new fullycovered self-expanding metal stent for the management of postesophagectomy strictures. Dig Endosc 2011; 23: 187-9.

5 Osaka Y, Shinohara M, Hoshino S, Ogata T, Takagi Y, Tsuchida A, et al. Phase II study of combined chemotherapy with docetaxel, CDDP and 5-FU for highly advanced esophageal cancer. Anticancer Res 2011; 31: 633-8.

6 Spigel DR, Greco FA, Meluch AA, Lane CM, Farley C, Gray JR, et al. Phase I/II trial of preoperative oxaliplatin, docetaxel, and capecitabine with concurrent radiation therapy in localized carcinoma of the esophagus or gastroesophageal junction. J Clin Oncol 2010; 28: 2213-9.

7 Ma Y, Ding Z, Qian Y, Wan YW, Tosun K, Shi X, et al. An integrative genomic and proteomic approach to chemosensitivity prediction. Int $J$ Oncol 2009; 34: 107-15.

8 Marusyk A, Polyak K. Tumor heterogeneity: causes and consequences. Biochim Biophys Acta 2010; 1805: 105-17.

9 Watkins JM, Zauls AJ, Kearney PL, Shirai K, Ruppert BN, Harper JL, et al. Toxicity, response rates and survival outcomes of induction cisplatin and irinotecan followed by concurrent cisplatin, irinotecan and radiotherapy for locally advanced esophageal cancer. Jpn J Clin Oncol 2011; 41: 334-42.

10 Brown E, Markman M. Tumor chemosensitivity and chemoresistance assays. Cancer 1996; 77: 1020-5.

11 Kurbacher CM, Cree IA. Chemosensitivity testing using microplate adenosine triphosphate-based luminescence measurements. Methods Mol Med 2005; 110: 101-20.

12 Qi CJ, Ning YL, Zhu YL, Min HY, Ye H, Qian KQ. In vitro chemosensitivity in breast cancer using ATP-tumor chemosensitivity assay. Arch Pharm Res 2009; 32: 1737-42.

13 Konecny G, Crohns C, Pegram M, Felber M, Lude S, Kurbacher C, et al. Correlation of drug response with the ATP tumorchemosensitivity assay in primary FIGO stage III ovarian cancer. Gynecol Oncol 2000; 77: 258-63.

14 Sharma S, Neale MH, Di Nicolantonio F, Knight LA, Whitehouse PA, Mercer SJ, et al. Outcome of ATP-based tumor chemosensitivity assay directed chemotherapy in heavily pre-treated recurrent ovarian carcinoma. BMC Cancer 2003; 3: 19.

15 Whitehouse PA, Knight LA, Di Nicolantonio F, Mercer SJ, Sharma $\mathrm{S}$, Cree IA, et al. Heterogeneity of chemosensitivity of colorectal adenocarcinoma determined by a modified ex vivo ATP-tumor chemosensitivity assay (ATP-TCA). Anticancer Drugs 2003; 14: 369-75.

16 Chou TC, Talalay P. Quantitative analysis of dose-effect relationships: the combined effects of multiple drugs or enzyme inhibitors. Adv Enzyme Regul 1984; 22: 27-55.

17 Whitehouse PA, Mercer SJ, Knight LA, Di Nicolantonio F, O'Callaghan A, Cree IA, et al. Combination chemotherapy in advanced gastrointestinal cancers: ex vivo sensitivity to gemcitabine and mitomycin C. Br J Cancer 2003; 89: 2299-304.

18 Kurbacher CM, Bruckner HW, Cree IA, Kurbacher JA, Wilhelm L, Pöch $\mathrm{G}$, et al. Mitoxantrone combined with paclitaxel as salvage therapy for platinum-refractory ovarian cancer: laboratory study and clinical pilot trial. Clin Cancer Res 1997; 3: 1527-33.
19 Sumantran VN. Cellular chemosensitivity assays: an overview. Methods Mol Biol 2011; 731: 219-36.

20 Glaysher S, Cree IA. Cell sensitivity assays: the ATP-based tumor chemosensitivity assay. Methods Mol Biol 2011; 731: 247-57.

21 von Bueren AO, Oehler C, Shalaby T, von Hoff K, Pruschy M, Seifert B, et al. C-MYC expression sensitizes medulloblastoma cells to radio- and chemotherapy and has no impact on response in medulloblastoma patients. BMC Cancer 2011; 11: 74.

22 Wang X, Yan SK, Dai WX, Liu XR, Zhang WD, Wang JJ. A metabonomic approach to chemosensitivity prediction of cisplatin plus 5-fluorouracil in a human xenograft model of gastric cancer. Int J Cancer 2010; 127: 2841-50.

23 Di Nicolantonio F, Knight LA, Di Palma S, Sharma S, Whitehouse PA, Mercer SJ, et al. Ex vivo characterization of XR11576 (MLN576) against ovarian cancer and other solid tumors. Anticancer Drugs 2004; 15: 849-60.

24 Doerler M, Hyun J, Venten I, Potthoff A, Bartke U, Serova K, et al. Does chemosensitivity-assay-directed therapy have an influence on the prognosis of patients with malignant melanoma stage IV? A retrospective study of 14 patients with malignant melanoma stage IV. Eur J Med Res 2007; 12: 497-502.

25 Mercer SJ, Somers SS, Knight LA, Whitehouse PA, Sharma S, Di Nicolantonio F, et al. Heterogeneity of chemosensitivity of esophageal and gastric carcinoma. Anticancer Drugs 2003; 14: 397-403.

26 Michalski CW, Erkan M, Sauliunaite D, Giese T, Stratmann R, Sartori $\mathrm{C}$, et al. Ex vivo chemosensitivity testing and gene expression profiling predict response towards adjuvant gemcitabine treatment in pancreatic cancer. Br J Cancer 2008; 99: 760-7.

27 Parker KA, Glaysher S, Polak M, Gabriel FG, Johnson P, Knight LA, et al. The molecular basis of the chemosensitivity of metastatic cutaneous melanoma to chemotherapy. J Clin Pathol 2010; 63: 1012-20.

28 Glaysher S, Yiannakis D, Gabriel FG, Johnson P, Polak ME, Knight LA, et al. Resistance gene expression determines the in vitro chemosensitivity of non-small cell lung cancer (NSCLC). BMC Cancer 2009; 9: 300.

29 Tanaka N, Kimura H, Faried A, Sakai M, Sano A, Inose T, et al. Quantitative analysis of cisplatin sensitivity of human esophageal squamous cancer cell lines using in-air micro-PIXE. Cancer Sci 2010; 101: 1487-92.

30 Fujita Y, Hiramatsu M, Kawai M, Nishimura H, Miyamoto A, Tanigawa N. Histoculture drug response assay predicts the postoperative prognosis of patients with esophageal cancer. Oncol Rep 2009; 21: 499-505.

31 Kurbacher CM, Cree IA, Bruckner HW, Brenne U, Kurbacher JA, Müller $\mathrm{K}$, et al. Use of an ex vivo ATP luminescence assay to direct chemotherapy for recurrent ovarian cancer. Anticancer Drugs 1998; 9: 51-7.

32 Bhalla KN. Microtubule-targeted anticancer agents and apoptosis. Oncogene 2003; 22: 9075-86.

33 Bull JM, Tormey DC, Li SH, Carbone PP, Falkson G, Blom J, et al. A randomized comparative trial of adriamycin versus methotrexate in combination drug therapy. Cancer 1978; 41: 1649-57. 